\title{
CMOS image sensor with pseudorandom pixel Placement
}

\author{
Junichi Akita ${ }^{\text {a) }}$ \\ Dept. of Infomations and Systems Engineering, Kanazawa University \\ Kakuma, Kanazawa, Ishikawa 920-1192, Japan \\ a)akita@is.t.kanazawa-u.ac.jp
}

\begin{abstract}
The picture element (pixel) in conventional image sensors are placed in the form of a lattice for ease of implementation. Lattice placement of pixels intrinsically has directional singularity on the clarity of image representation, and the clarity is significantly dependent on the directions of the objects in the image. For example, horizontal lines are perfectly represented, while slanted lines have jagged edges.

In this paper, we describe the CMOS image sensor with the pseudorandom pixel placement that solves the problem of the directional singularity on the clarity of image representation, as well as its evaluation in terms of the clarity of image representation for slant lines.
\end{abstract}

Keywords: CMOS image sensor, jaggy, pixel placement, directional singularity, image clarity

Classification: Integrated circuits

\section{References}

[1] G. Meynants et al., "A 9 megapixel aps-size cmos image sensor for digital still photography," Proc. of 2005 IEEE Workshop on CCDs and AIS, pp. 246-249, June 2005.

[2] E. G. Stevens et al., " $8.3 \mathrm{mp}, 4.3$ " full-frame ccd with scaled lod and micro optics," Proc. of 2005 IEEE Workshop on CCDs and AIS, pp. 250-253, June 2005.

[3] M. F. Deering, "A photon accurate model of the human eye," ACM Transactions on Graphics, vol. 24, no. 3, pp. 649-658, March 2005.

[4] M. L. Hennelly et al., "The effect of age on the light scattering characteristics of the eye," Ophthal. Physiol. Opt., vol. 18, no. 2, pp. 197-203, Feb. 1998.

\section{Introduction}

The common ultimate purpose of the image sensors can be summarized as that of representing objects clearly, realistically. One of the most remarkable directions in developments of image sensors to achieve this purpose is to increase the resolution of imaging systems $[1,2]$. 
Although the picture element (pixel) in conventional image sensors are placed in the form of a lattice for ease of implementation, the lattice placement of pixels intrinsically has directional singularity on the clarity of image representation; in other words, the clarity is significantly dependent on the directions of the objects in the image. For example, horizontal lines are perfectly represented with no jaggies, while slanted lines have jagged edges, and these problems cannot be completely solved by increased number of pixels.

In this paper, we propose an idea for image sensors that can represent the clear images with fewer pixels based on the fact that represented images are ultimately "seen" by human eyes. The proposed image sensor has a pseudorandom pixel placement architecture so as to solve the directional singularity of the clarity of image representation. We also discuss our evaluation of its image representation characteristics based on the designed layout of CMOS image sensor with pseudorandom pixel placement.

\section{Pseudorandom pixel placement and image representation}

Although almost all the image sensors employ the lattice layout of pixels, the images represented by the image sensors are ultimately "seen" by human beings. We see the images with our eyes, particularly the retina in our eyes, where the photoreceptor cells are not placed regularly [3]. Because of this fact, human perceive the pixel placement of the conventional imaging systems as a "lattice" by us.

The clarity of the represented images using the lattice placement intrinsically depends on the direction of the objects in the image, which we call "directional singularity" on the clarity of image representation. For example, horizontal or vertical lines are perfectly represented as lines with no jaggies, while the slanted lines, especially those with smaller slopes, are represented with jaggy edges derived from the lattice approximation of slant lines. The effect of directional singularity on the clarity of image representation cannot be completely eliminated by increasing the resolution, because each jaggy is made up of a pair of pixels. In addition, the size of jaggies may changes dynamically in the movies, and our eye and brain perceive such temporal changes of jaggies with emphasis.

The effect of directional singularity on the clarity of image representation is caused by the mismatch between pixel placements in image sensors and the placement of the photoreceptor cells on the retina. Here, we propose a realistic idea for implementing pixel placement that prevents the negative effect of directional singularity on the clarity of image representation. The photo diode area in pixels performs as intrinsic interface in the image sensors for image capture. Figure 1 (a) illustrates four types of pixels whose photo diodes are placed at each corner in pixels, upper left, upper right, lower left, and lower right, respectively. The signal and power connections at pixels' boundaries are identical in order to implement correct electrical connection regardless of the sequence of pixel placement. Conventional CMOS image sensor with lattice pixel placement can be implemented by placing one of the 


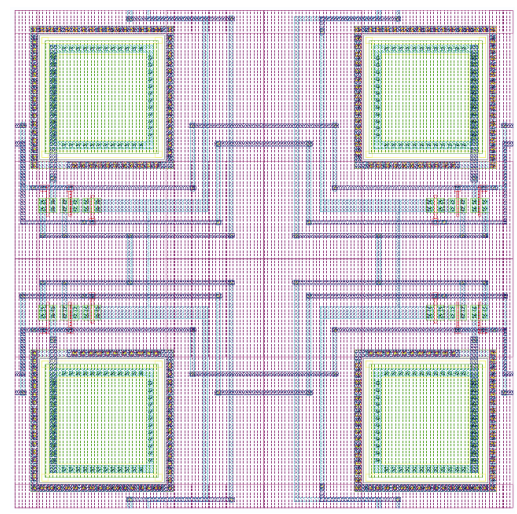

(a)

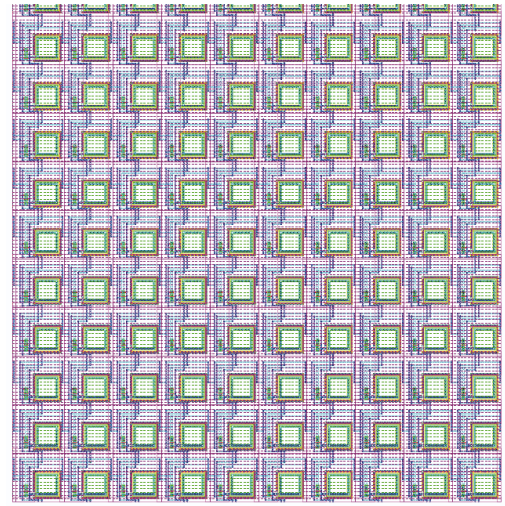

(b)

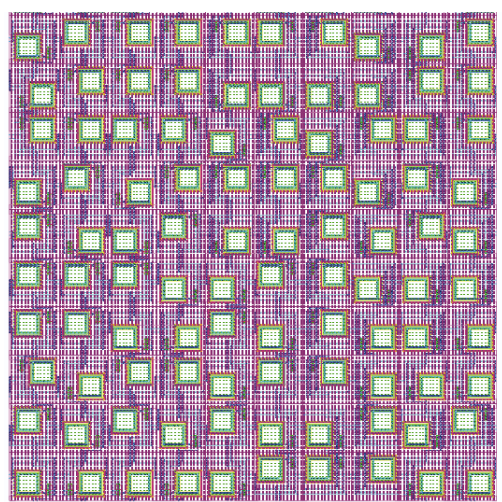

(c)

Fig. 1. Designed layout of CMOS image sensor. (a) Four types of unit pixels, and magnified $10 \times 10$ pixels of CMOS image sensors with (b) lattice placement and (c) pseudorandom placement, respectively.

unit pixels in a lattice layout.

Here, we assume randomly choosing one of the four types of unit pixel shown in Fig. 1(a), and putting it in the lattice positions, which we call "pseudorandom placement." The placement of the photo diodes is expected to be a good approximation of random placement, and also of the photoreceptor placement on the retina, and thus to eliminate the effect of directional singularity on image clarity that is caused by the lattice pixel placement applied in the conventional image sensors.

The advantages of using pseudorandom pixel placement described above in image sensor for better image clarity can be summarized as follows.

- The image clarity or jaggy size in the displayed images does not depend on the direction of lines (descreased effect of directional singularity on image clarity)

- The existing jaggies in the displayed images are expected to be so small that we do not perceive them in well-developed imaging systems 


\section{Design of CMOS Image Sensor with Pseudorandom Pixel Placement}

Figure 1 (a) shows the designed four types of unit pixels, whose photo diodes are located at upper left, upper right, lower left, and lower right corner, respectively using CMOS $0.18 \mu \mathrm{m}$ technology with five layers of metal. The pixel size is $14[\mu \mathrm{m}] \times s 14[\mu \mathrm{m}]$, and the photo diode size is $7[\mu \mathrm{m}] \times 7[\mu \mathrm{m}]$, with fill factor of $25 \%$.

We designed two types of CMOS image sensor whose photo diodes are located in lattice positions and pseudorandom positions.

The CMOS image sensor with lattice pixel placement is designed using one of the designed unit pixels in Fig. 1 (a), whose number of pixels is $100 \times$ 100. The part of the designed layout of placed pixels is shown in Fig. 1 (b) for $10 \times 10$ pixels. The unit pixel whose photo diode is placed at upper right corner is placed in the lattice manner. In this paper, the details of the other circuit components in whole image sensor, such as row decoders, column selectors with CDS (correlated double sampling) circuits, and output buffers or A/D converters, are not discussed, since we focused on the evaluation of image representation characteristics derived from the variation of photo diode placement, discussed on section 4 .

The CMOS image sensor with pseudorandom pixel placement is also designed using the designed unit pixels in Fig. 1 (a), whose number of pixels is $100 \times 100$. The randomly chosen unit pixels in four types of unit pixels are placed for implementing pseudorandom placement of photo diodes. The part of the designed layout of placed pixels is also shown in Fig. 1 (c) for $10 \times 10$ pixels.

\section{Evaluation of Designed CMOS Image Sensor}

We carried out the evaluation of the characteristics of the designed CMOS image sensor, in terms of the eliminating effect of jaggies for slant lines.

A slant line with slope of 3 degrees and width of 5 pixel size is assumed to be projected onto two designed CMOS image sensors, and the 'value' of photo diode is set as 1 or 0 according to whether it is included in the projected line or not, respectively. Figure 2 shows the captured images of line with slope of 3 degrees by two types of CMOS image sensors with lattice and pseudorandom pixel placements as shown in Fig. 1(b) and Fig. 1 (c).

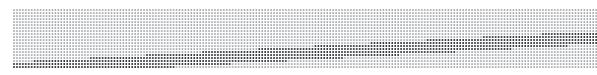

(a)

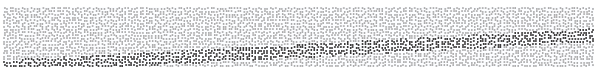

(b) 


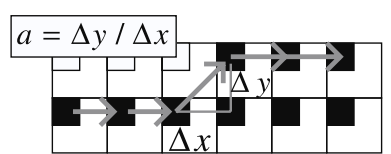

(a)

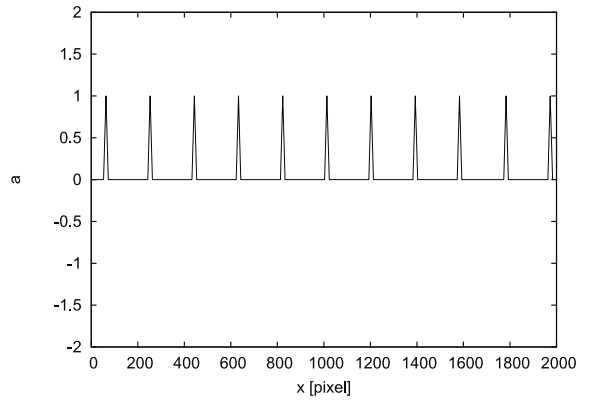

(b)

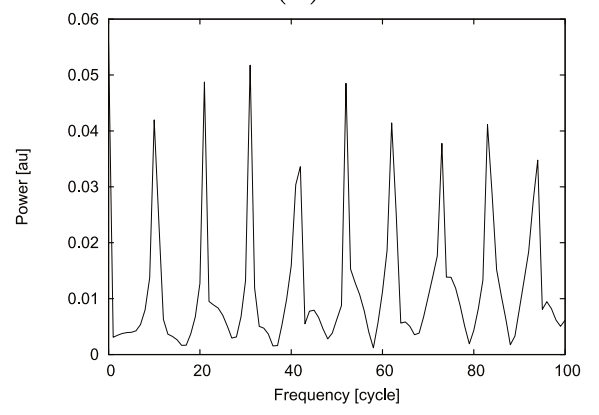

(d)

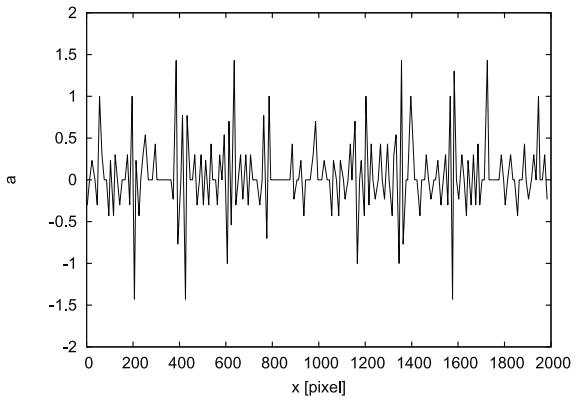

(c)

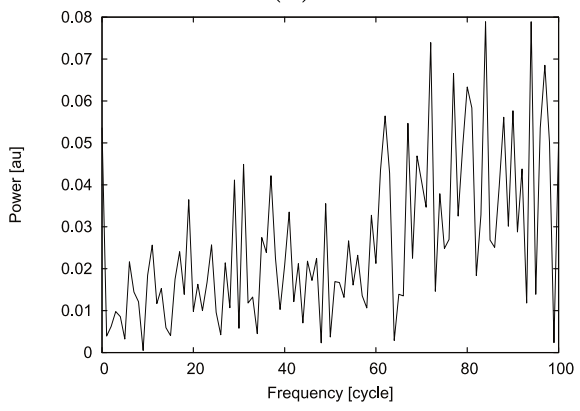

(e)

Fig. 3. (a)Definition of local slope of line edge, $a$, and the trends of $a$ for (b) Lattice, (c) Pseudorandom, as well as their spectrum of $a$ trends for (d) Lattice, (e) Pseudorandom.

In Fig. 2, the photo diodes with value of 0 are indicated as gray for ease of viewing.

The viewing angle of one pixel is approximately 0.04 degrees when you see Fig. 2 viewed at the distance of $40 \mathrm{~cm}$ from the paper. The viewing angle that our eyes can perceives is down to approximately 0.1 degrees [4], and thus, the jaggies with larger than 2 or 3pairs of virtual pixels, whose view angle is within that range we can perceived.

Here, we consider the local slope of photo diodes composing the line, $a=$ $\Delta y / \Delta x$, as shown in Fig. 3 (a). The line in the lattice placement has $a$ of 0 for most pixels, and $a$ of 1 for some pixels with an interval according to the angle of the slope. This drastic change of $a$ for the particular interval becomes a jaggy with lower spatial frequency that can be perceived by our eyes. In other words, the spatial frequency component of $a$ in the image according to this change of $a$ stands out compared with other spatial frequency components in the spatial spectrum of $a$.

The trends of local slope $a$ for lines represented in both Fig. 2 (a) and Fig. 2 (b) are shown in Fig. 3(b) and Fig. 3(c), respectively. The spatial power spectrum of both trends of local slope $a$ are also shown in Fig. 3 (d) and Fig. 3(e), respectively. In Fig. 3(d), lattice placement, the spatial fre- 
quency component of 10 cycles, or the interval of 20 virtual pixels present a sharp spectrum, as well as its higher harmonic components. Because the first spatial frequency component of 10 cycles exists within the range that our eyes perceive, we recognize it as a serious jaggy.

On the other hand, a similar component exists for pseudorandom placement as shown in Fig. 3 (e); however, the other spatial frequency components with large amplitude appear near this component. In other words, the spatial spectrum component of 10 cycle does not stand apart from the whole spatial spectrum compared with nearby components, and thus, our eyes do not perceive the jaggy.

The ratio of the spatial power spectrum components amplitude of 6 cycles against the nearby components is estimated as 1:10 for lattice placement in Fig. 3 (d), but is 1:1 for pseudorandom in Fig. 3 (e).

\section{Conclusions}

Directional singularity negatively affects image representation clarity. In this paper, we proposed the pseudorandom pixel placement for image sensors that eliminates the effect of directional singularity on image clarity. We designed the practical CMOS image sensors with two types of active area placements, conventional lattice and pseudorandom proposed. We also evaluated the characteristics of pseudorandom placement in terms of spatial spectrum and representation of line, and our results show that pseudorandom placement significantly improves image representation clarity, especially that for slant lines. 